\title{
Comparative Analysis of Electron Density and Electric Field Fluctuations in Highly Active Aurora Observed by Sounding Rocket S-310JA-12
}

\author{
Hirotaka MORI ${ }^{1}$, Eiichi SAgawa ${ }^{2}$, Tadahiko Ogawa ${ }^{2}$, Toshio Ogawa ${ }^{3}$, \\ Hisao YAMAGISHI ${ }^{4}$, and Hiroshi FUKUNISHI ${ }^{5}$ \\ ${ }^{1}$ Hiraiso Solar Terrestrial Research Center, Radio Research Laboratory, \\ Nakaminato-shi, Ibaraki 311-12, Japan \\ ${ }^{2}$ Radio Research Laboratory, Koganei-shi, Tokyo 184, Japan \\ ${ }^{3}$ Department of Physics, Kochi University, Kochi 780, Japan \\ ${ }^{4}$ National Institute of Polar Research, Itabashi-ku, Tokyo 173, Japan \\ ${ }^{5}$ Faculty of Science, Tohoku University, Sendai 980, Japan
}

(Received November 10, 1987; Revised February 25, 1988)

The S-310JA-12 rocket was launched toward geomagnetic north from Syowa Station, Antarctica, during a strong substorm. A Faraday cup aboard the rocket measured electron density and its fluctuations up to $5 \mathrm{kHz}$, together with total flux of electrons having energies above $105 \mathrm{eV}$, while a pair of probes measured DC electric fields and electric field fluctuations in the $5-220 \mathrm{~Hz}$ range. During ascent, the Faraday cup detected burst-like fluctuations between $140 \mathrm{~km}$ and $200 \mathrm{~km}$ in altitude, which are believed to have been artificially generated around the rocket. Around the altitude of $220 \mathrm{~km}$, quasi-sinusoidal waves of about $150 \mathrm{~Hz}$ were observed by both instruments. From the cross-correlation analysis, they are found to be right-handed, circularly or elliptically poralized waves. During descent, the rocket passed through a highly active aurora and observed strong ELF fluctuations. The Faraday cup measurements suggest that the rocket was charged to several volts by the precipitating electrons.

\section{Introduction}

During an auroral display, wave-particle interactions in the ionosphere play a principal role in the acceleration and scattering of precipitating electrons. In order to clarify these processes, simultaneous observations of waves and particles on a sounding rocket were undertaken. Previous observations of ELF waves were made by a Faraday cup and a pair of (double) probes onboard the S-310JA-7 rocket. Results from this flight showed that this combination of instruments is very effective for identifying mode of the observed plasma waves (MORI et al., 1985). In order to obtain another set of data, we again installed the same combination on the S-310JA-11 and S-310JA-12 rockets (YAMAGISHI and FUKUNISHI, 1985). This paper presents the results only from the S-310JA-12 rocket experiment. 


\section{Instrumentation}

The Faraday cup consists of four grids (G1 to G4) and one collector. All electrodes are plated with gold. Its diameter and height are 100 and $30 \mathrm{~mm}$, respectively. As shown in Fig. 1, appropriate DC voltages are applied to the grids and collector so as to collect electrons. First grid (G1) biased at $+3 \mathrm{~V}$ relative to rocket body measures the electron DC current (PWN-D). Second grid (G2) biased at $+15 \mathrm{~V}$ is used to measure the $\mathrm{AC}$ component of electron current. Output from the G2 is divided into two frequency bands; one is the low-frequency band from $5 \mathrm{~Hz}$ to $330 \mathrm{~Hz}$ (PWN-L), and another is the high-frequency band from 0.1 to $5 \mathrm{kHz}(\mathrm{PWN}-\mathrm{W})$ which is sent to the ground via wide-band telemetry (WBTM). The wide-band telemetry link is time-shared with another instrument for ELF-VLF wave measurement (PWL), with $3 \mathrm{~s}$ out of every $7 \mathrm{~s}$ period being allocated to the PWN-W. The collector and G4 are biased at -90 and $-105 \mathrm{~V}$, respectively, so that the collector responds to the electrons with energies greater than $105 \mathrm{eV}$, while $\mathrm{G} 4$ serves as a suppressor grid (PWN-F). Numbers followed by the character ch. in the parentheses in the right part of the figure show the analog telemeter IRIG channel numbers to be used. The Faraday cup was installed in the forward section of the rocket.

Two probes, separated by $2.12 \mathrm{~m}$, were extended perpendicular to the rocket body and were employed to measure DC electric fields (AEF-D) and AC electric field fluctuations in a frequency range from 5 to $220 \mathrm{~Hz}$ (AEF-L). Details of this instrument was described by OGAWA et al. (1981).

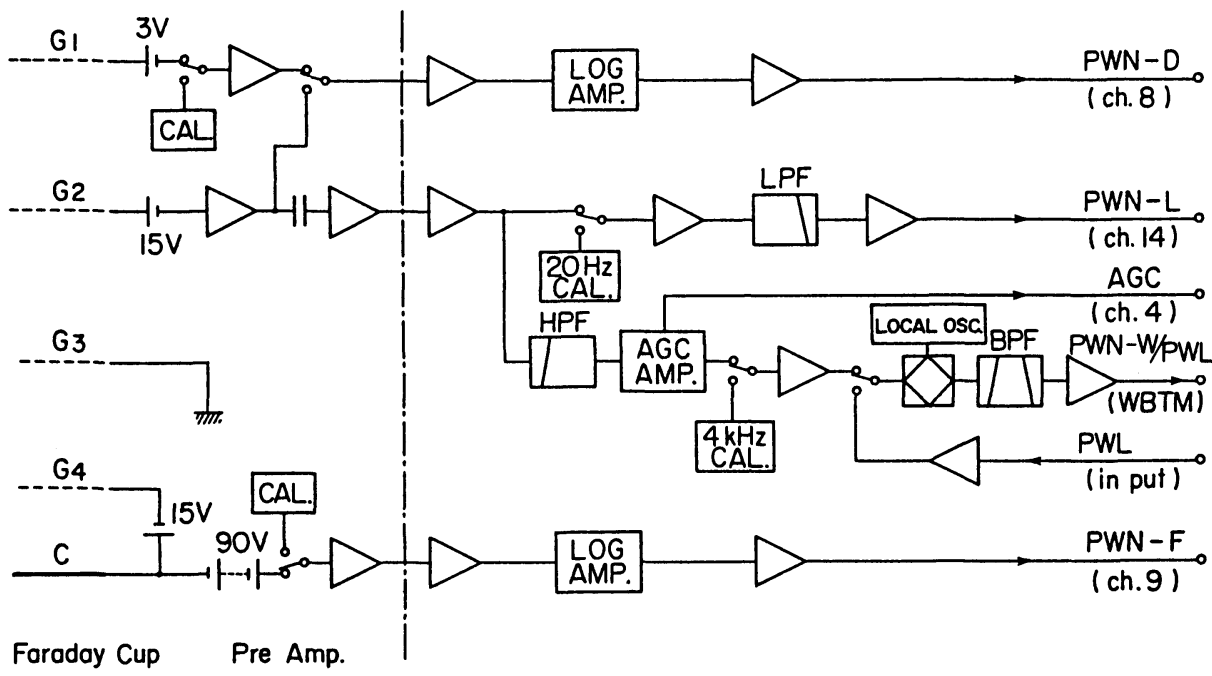

Fig. 1. Block diagram of the PWN-D, $-\mathrm{L},-\mathrm{W}$ and $-\mathrm{F}$ payloads aboard the S-310JA-12 rocket. 


\section{Observations and Discussions}

The S-310JA-12 rocket was launched in the geomagnetic northward (equatorward) direction at 19:35:39 UT on July 12, 1985, and flew through highly active aurora during a strong substorm (EJIRI et al., 1988).

Figure 2 shows the time variations of the PWN-D, PWN-F, PWN-L $\mathrm{L}_{\mathrm{rms}}$, and AEF- $\mathrm{L}_{\text {rms }}$ data during the flight, where subscripts rms stand for root-mean-square average of the amplitude of the signal filtered out with the $40 \mathrm{~Hz}$ high-pass filter. The PWN- $\mathrm{L}_{\text {rms }}$ value is normalized to the corresponding PWN-D current. Because the PWN-D current was saturated during the 66-90 s time interval $(95-129 \mathrm{~km})$ due to the high electron density $\left(>5 \times 10^{5} \mathrm{~cm}^{-3}\right)$, the PWN-L $\mathrm{L}_{\mathrm{rms}}$ is normalized to the PWN-D current which is estimated from the electron density data obtained by the onboard impedance probe (NEI) for this time period (TAKAHASHI et al., 1988), where the ratio of PWN-D current and the electron density within the time period is assumed constant and equal to the value of just before and after the time period. The PWN-F current increased abruptly at about $70 \mathrm{~s}(101 \mathrm{~km})$, which indicates that the rocket

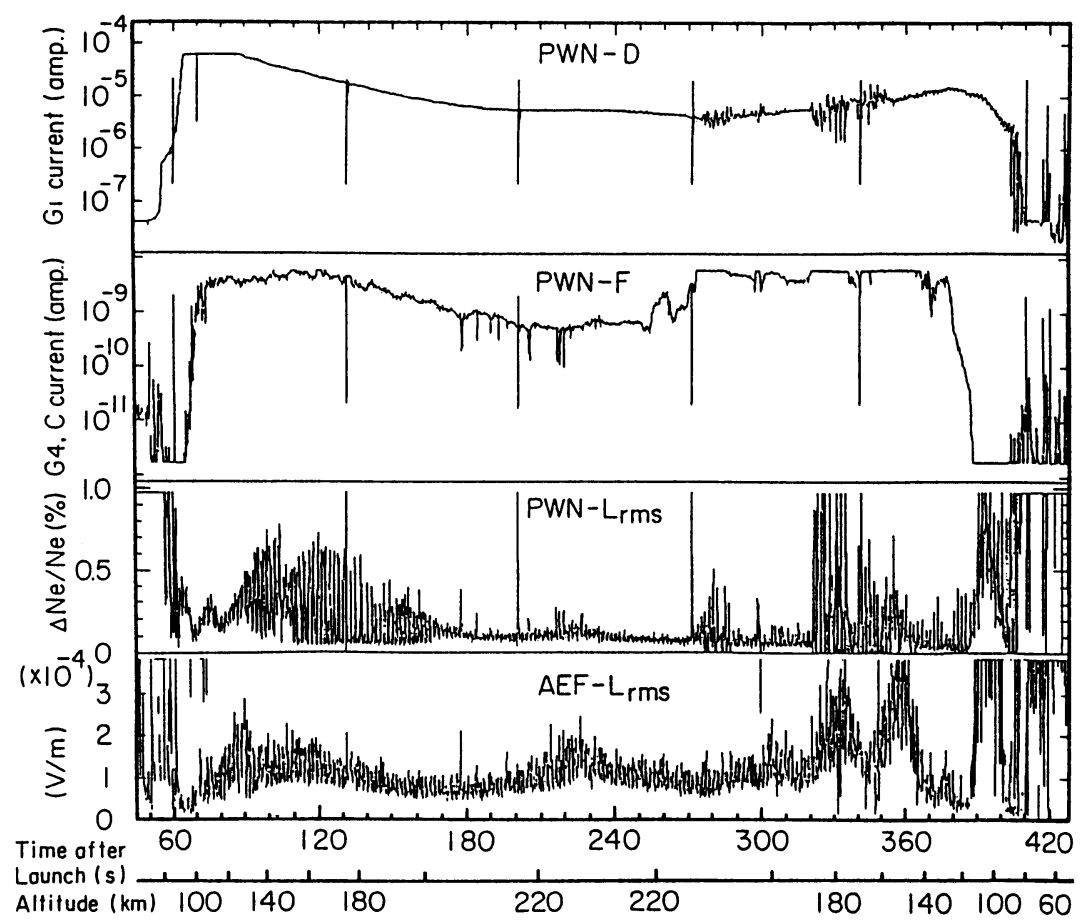

Fig. 2. Time variations of the PWN-D (upper) and PWN-F (second) signals, and root-mean-square averages of the PWN-L (third) and AEF-L (bottom) signals. 
encountered the aurora. The rocket re-entered the active aurora at about $272 \mathrm{~s} \mathrm{(216}$ $\mathrm{km})$, and the PWN-F current showed saturation effects until $367 \mathrm{~s}(141 \mathrm{~km})$. During this period, the PWN-D current also fluctuated considerably.

Temporal fluctuations were also observed in the PWN-L $\mathrm{L}_{\mathrm{rms}}$ and AEF- $\mathrm{L}_{\mathrm{rms}}$ data. The periods of signal enhancements are discussed below.

\subsection{Fluctuations during the ascent}

Figure 3 shows the PWN-L $\mathrm{L}_{\text {rms }}$ and AEF- $\mathrm{L}_{\text {rms }}$ data during the 61-156 s (87-196 $\mathrm{km}$ ) interval. According to the $\mathrm{PWN}-\mathrm{L}_{\mathrm{rms}}$ records, the Faraday cup observed continuous fluctuations with amplitudes less than $0.4 \%$ in the $E$-region $(90-130 \mathrm{~km})$. Although the $\mathrm{PWN}-\mathrm{L}_{\mathrm{rms}}$ is amplitude average of the PWN-L data within the frequency range of $40-330 \mathrm{~Hz}$, spectral analysis of the PWN-W $(0.1-5 \mathrm{kHz})$ data for the same region shows that most of the power resided in the $2-5 \mathrm{kHz}$ range. Therefore, the amplitude integrated between $40 \mathrm{~Hz}$ and $5 \mathrm{kHz}$ must have been larger than $0.4 \%$. Above $130 \mathrm{~km}$, the fluctuations gradually changed their shape and appeared intermittently with maximum amplitudes around $120 \mathrm{~s}(165 \mathrm{~km})$. Occurrence of these burst-like fluctuations is found to be well organized by the rocket spin phase as shown in Fig. 4. Plotted here is the spin phase dependence of the enhanced density fluctuations from 90 to $165 \mathrm{~s}(128-200 \mathrm{~km})$. The spin phase angle is taken to be zero when the output from the MGF-H sensor for measuring the radial component of the geomagnetic field with the rocket fixed coordinates is maximum, which means that the sensor points the same direction as the perpendicular component vector of the geomagnetic field vector to the rocket axis vector (TOHYAMA et al., 1988). Also shown in the figure, by white vertical lines, are the phase angles for which either of the double probes is inside the rocket wake. It is determined from the AEF-D data by a pair of characteristic peaks with opposite polarities appearing every spin period which are caused by drastic change of plasma condition surrounding the probe within the wake. It can be seen from the figure that these fluctuations are strongly connected with the rocket spin phase after about $130 \mathrm{~s}$. On the other hand, the AEF- $\mathrm{L}_{\mathrm{rms}}$ data shown in Fig. 3 does not exhibit such burst-like behavior during this time period.

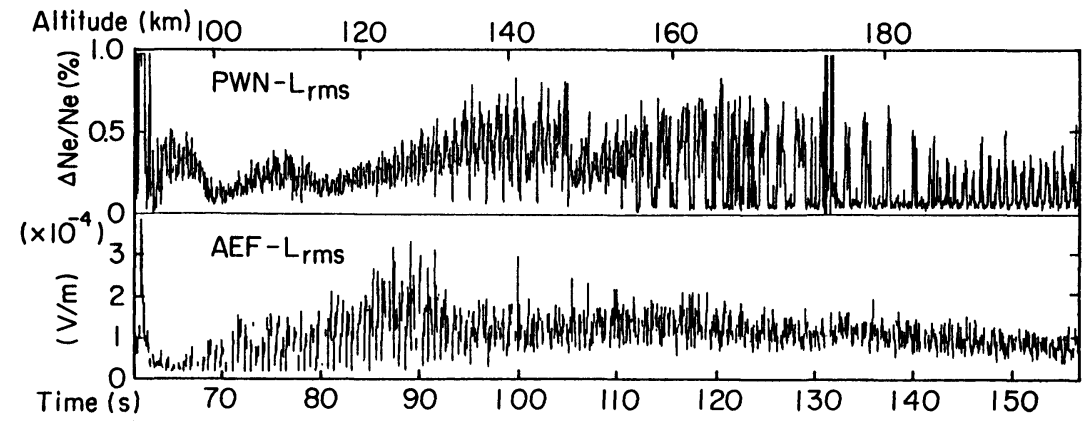

Fig. 3. Temporal variations of the PWN-L (upper) and AEF-L (lower) amplitudes during the ascent. 


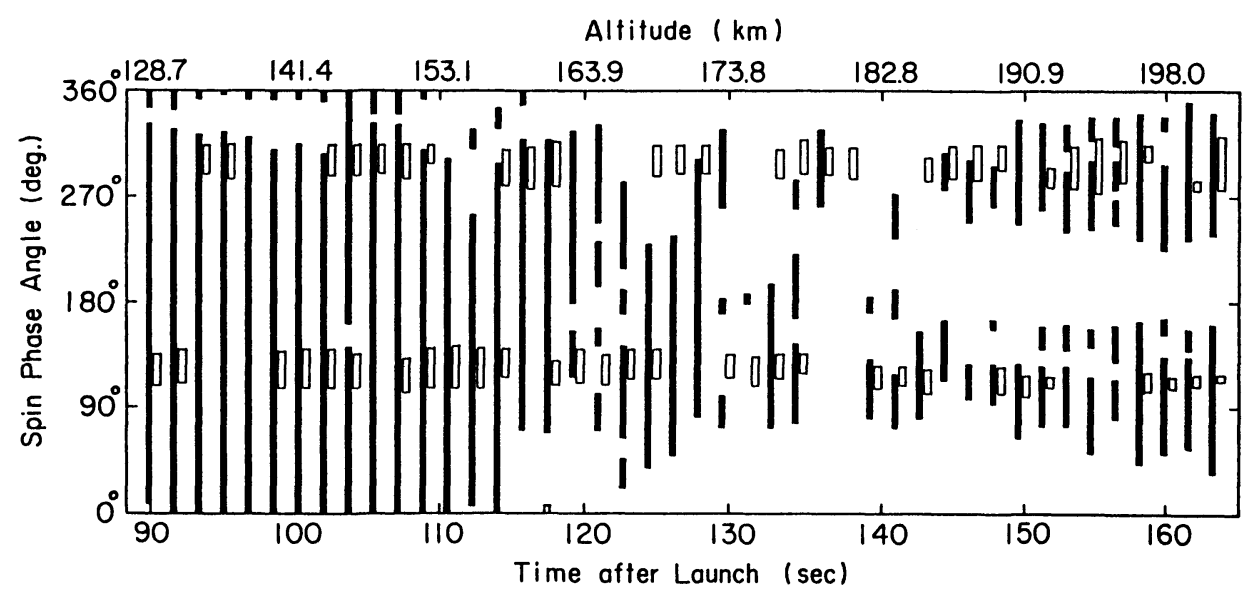

Fig. 4. Occurrence of the burst-like fluctuations ( $)$ plotted as a function of the rocket spin phase angle. (־) denotes times when one of the double probes were inside the wake of the rocket.

Figure 5(a) shows the phase angle distribution of the occurrence frequency of the burst-like fluctuations from 140 to $165 \mathrm{~s}(183-200 \mathrm{~km})$. Note that the distribution has two clear types of peaks (I and II), and each type having two peaks separated by $180^{\circ}$. This suggests that these fluctuations were generated by two pairs of antennas rotating around the rocket. In this case, disturbances caused by the antennas could be detected by the Faraday cup mounted at the top of the rocket when the antennas directed along the rocket velocity vector. Since the rocket flew close to the geomagnetic northward direction, this happened when the antennas were in the geomagnetic meridian plane. Therefore, phase dependent perturbations are expected at the setting angles of the related antennas which are measured anti-spin-wisely from the MGF-H sensor. Figure 5(b) shows the orientation diagram of antennas and sensors radially extended from the rocket body. Referring to the relative azimuthal angles of the AEF double probes, MGF-H sensor and PWH dipole antennas for measuring HF waves and to the rocket spin sense, the correspondence between the phase angle difference between two types (I and II) and the azimuthal separation of three instruments strongly supports the assumption that these fluctuations are induced by interactions of these sensors with the ambient plasma.

\subsection{Quasi-sinusoidal waves observed near apogee}

Figure 6 shows the dynamic spectra of the PWN-L and AEF-L data during the 29-413 s time interval obtained by the Maximum Entropy Method (MEM) analysis. It must be noted that the broad band spectra extending from $0 \mathrm{~Hz}$ to about $200 \mathrm{~Hz}$ which appear every $10.7 \mathrm{~s}$ are not caused by natural waves but caused by interferences from other onboard instruments. Near apogee (210-240 s), spectral intensity enhancements were observed at frequencies centered around $150 \mathrm{~Hz}$ in both spectra. 
(a)

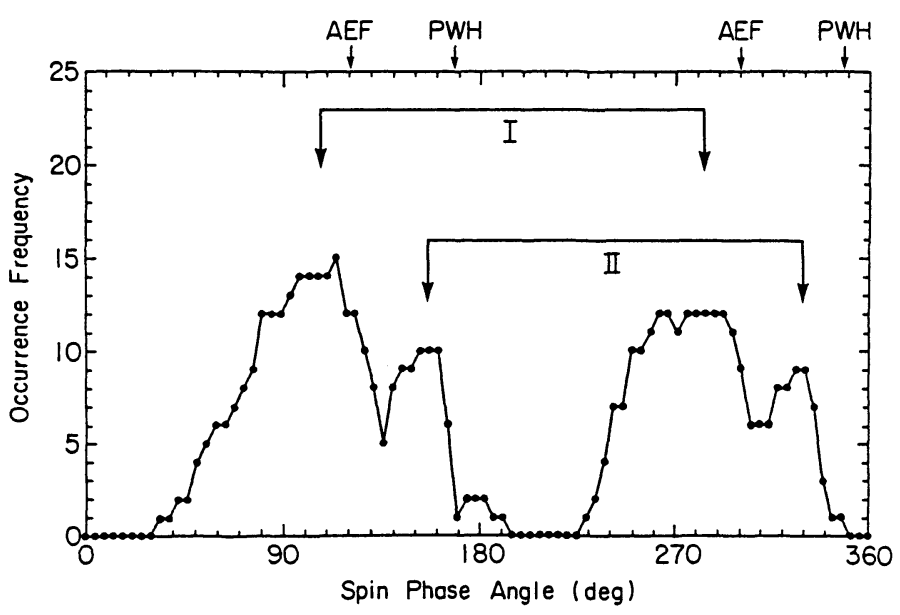

(b)

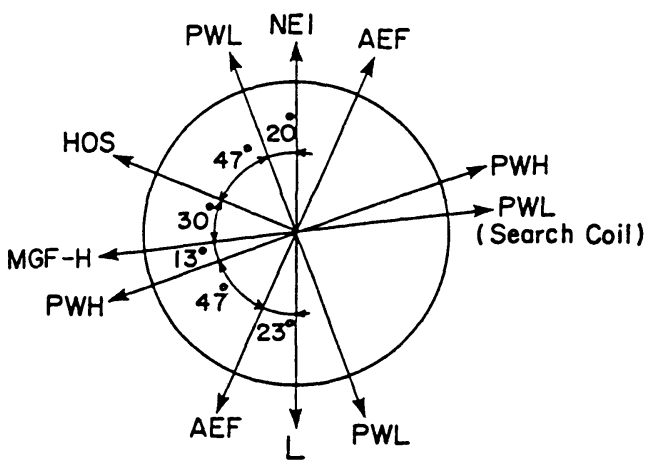

Fig. 5. (a) Phase angle distribution of the occurrence frequency of the burst-like fluctuations during 140-165 s. Two pairs of two arrows labeled AEF and PWH indicate the setting angles of these sensors with respect to the MGF-H sensor. (b) Orientation diagram of an antennas and sensors of S-310JA-12 rocket.

Raw data of a 0.3 s interval taken during one of these events is shown in Fig. 7. Intense quasi-sinusoidal waves in both the Faraday cup and double probe data are clearly observable.

In order to compare these two data sets more quantitatively, a cross-correlation analysis was performed. Shown in Fig. 8 in the top panel are the correlation function amplitudes as a function of lag time every $25 \mathrm{~ms}$ during the 224.1-227.3 s time interval. This PWN-L with AEF-L correlation was performed with 64 ms data samples. Lag times of AEF-L data samples with respect to PWN-L ones ranging from -20 to $20 \mathrm{~ms}$ were used as indicated on the ordinate in Fig. 8. The lower panel plots the lag times at 


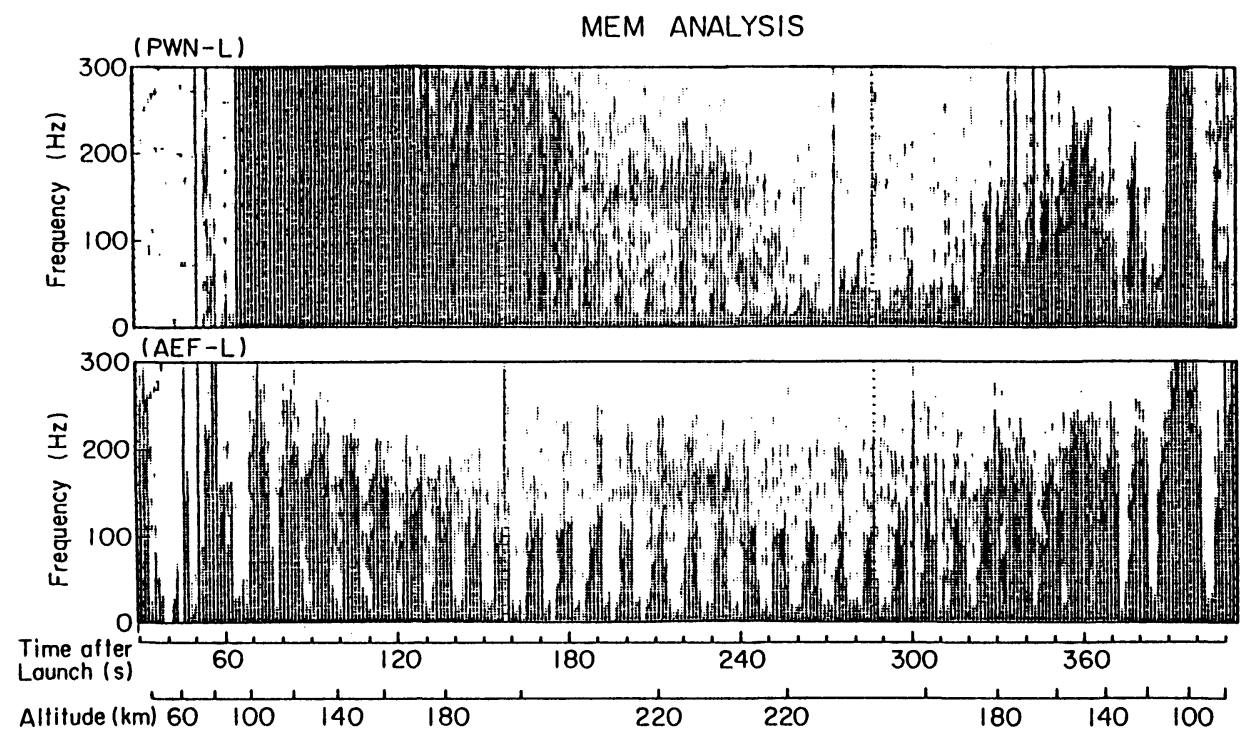

Fig. 6. Dynamic spectra of the PWN-L (upper) and AEF-L (lower) data.

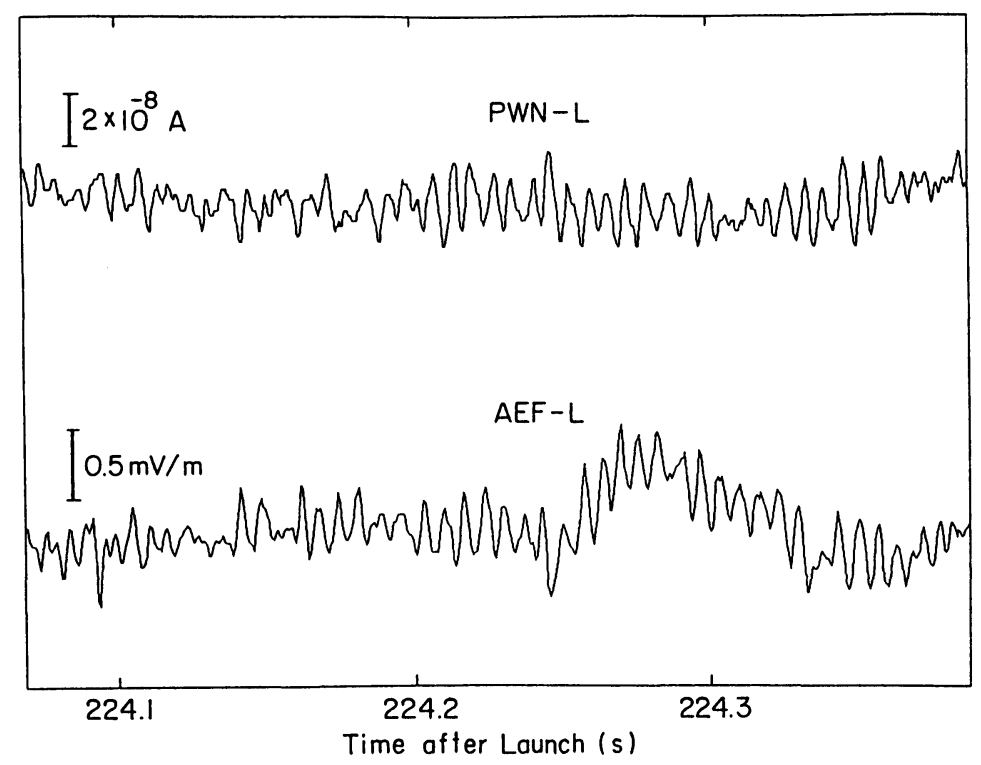

Fig. 7. Raw data obtained by the PWN-L and AEF-L during 224.1-224.4 s. 


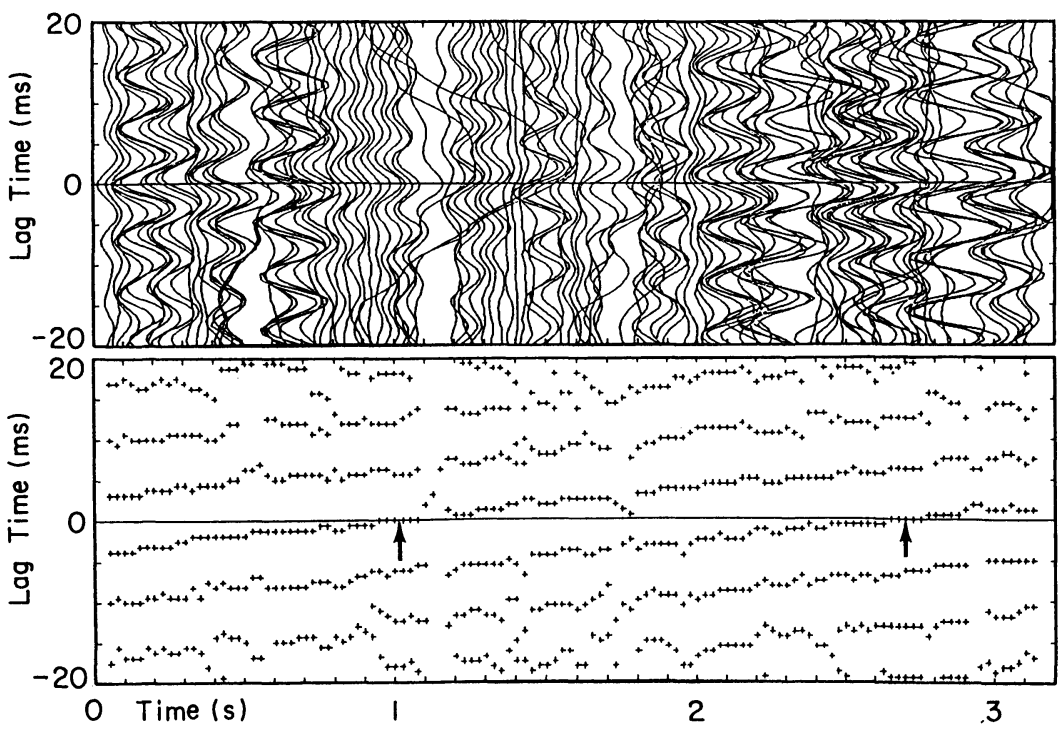

Fig. 8. Cross-correlation functions of the PWN-L and AEF-L data (upper) during 224.1-227.3 s, and locations of correlation peaks (lower). The two arrows in the lower panel indicate the times when the two signals were in-phase. Note that the period between the two arrows coincides with the rocket spin period (1.68 s).

which the cross-correlation functions shown in the upper panel reaches a peak. Note that the phases of cross-correlation functions shift almost linearly with time, and that the time interval for a shift of $360^{\circ}$ coincides exactly with the rocket spin period (1.68 s), as indicated by two arrows in the figure. This means that the frequency observed by the double probes is less, by the rocket spin frequency, than that observed by the Faraday cup. These data are consistent with the observations of a wave with an electric field vector which rotated in the rocket spin direction. Considering that the double probes rotate clockwise as looking forward with the spin frequency, and that the geomagnetic field points upward, it is concluded that the electric field component of the observed quasi-sinusoidal wave was right-hand circularly or elliptically polarized. Similar quasi-sinusoidal waves with frequencies of $38-45 \mathrm{~Hz}$ and with the same sense of rotation as the rocket results were previously observed by the S-310JA7 rocket (OGAWA et al., 1981; MORI et al., 1985).

Since it is unlikely that a rocket moving in plasma would generate artificially such polarized wave, it is reasonable to conclude that the waves were generated naturally. To identify the generation mechanism of the waves, it is important to identify their propagation mode. Natural waves, in general, can be classified into two categories, i.e., electrostatic and electromagnetic waves. Electrostatic waves are characterized by longitudinal electric field and electron density variations with linear polarization. The circular or elliptical polarization properties of the waves obtained in 
our experiment clearly indicate that they do not belong to the electrostatic wave category. However, the Faraday cup data (PWN-L) imply that the waves had electron density variations along with electric field variations. This suggests that the waves can not be simply identified as circularly polarised electromagnetic waves propagating parallel to the geomagnetic field lines, since this mode, the ordinary mode, does not have large amplitude electron density variations associated with it. On the other hand, extraordinary electromagnetic waves which propagate almost perpendicular to the geomagnetic field have elliptically polarized electric fields, and can be accompanied by electron density variations. Therefore, this mode seems to be the most plausible explanation for the observed waves. Further analysis including the determination of the propagation angle of the waves is required before a definite conclusion may be reached.

\subsection{Fluctuations in an active aurora during the descent}

The rocket passed through a highly active aurora on descent, and during the traversal of the aurora the PWN-F data was often saturated. Simultaneously the PWN-D signal showed large fluctuations. Amplitudes of the PWN-L and AEF-L signals also increased. The upper panel of Fig. 9 shows the temporal variations of the PWN-F, PWN-D and AEF-D signals from 269 to $397 \mathrm{~s}(217-101 \mathrm{~km})$. The ordinates are arbitrarily set for comparison. The lower panel depicts a magnified view of a part of the upper panel. Note that the PWN-F data during the period shown in the lower panel were almost saturated. The staircase-like trace of the data is caused by the digitization process ( 8 bit analog-to-digital conversion). The broken line in the lower panel indicates the quiet PWN-D signal level before and after the traverse of the aurora.

It can be seen that the PWN-D current fluctuations were strongly associated with the PWN-F variations. Particularly, the PWN-D levels in the shaded regions drop to nearly one tenth of the quiet level given by the broken line. Since, according to the NEI measurement, the local electron density varied at most by a factor of two, it is likely that the electric potential of the G1 of the Faraday cup was temporarily below the local plasma potential, and, therefore, most of the thermal electrons could not reach the Faraday cup. Such phenomena may occur if the potential of the rocket surface was lowered by more than a few electron volts from the normal plasma potential by charging due to a large flux of high energy electrons. The AEF-D data also showed large amplitude variations most peaks and dips of which seem to appear in coincidence with the PWN-F peaks. This may reflect a complicated spatial distribution of electric fields in the region. However, unbalanced charging of each of the double probes must also be considered. Further study of the potential of the rocket using the in-situ auroral particle observations is required before the rocket potential may be determined.

\section{Conclusion}

The S-310JA-12 rocket was launched into highly active aurora during a strong 


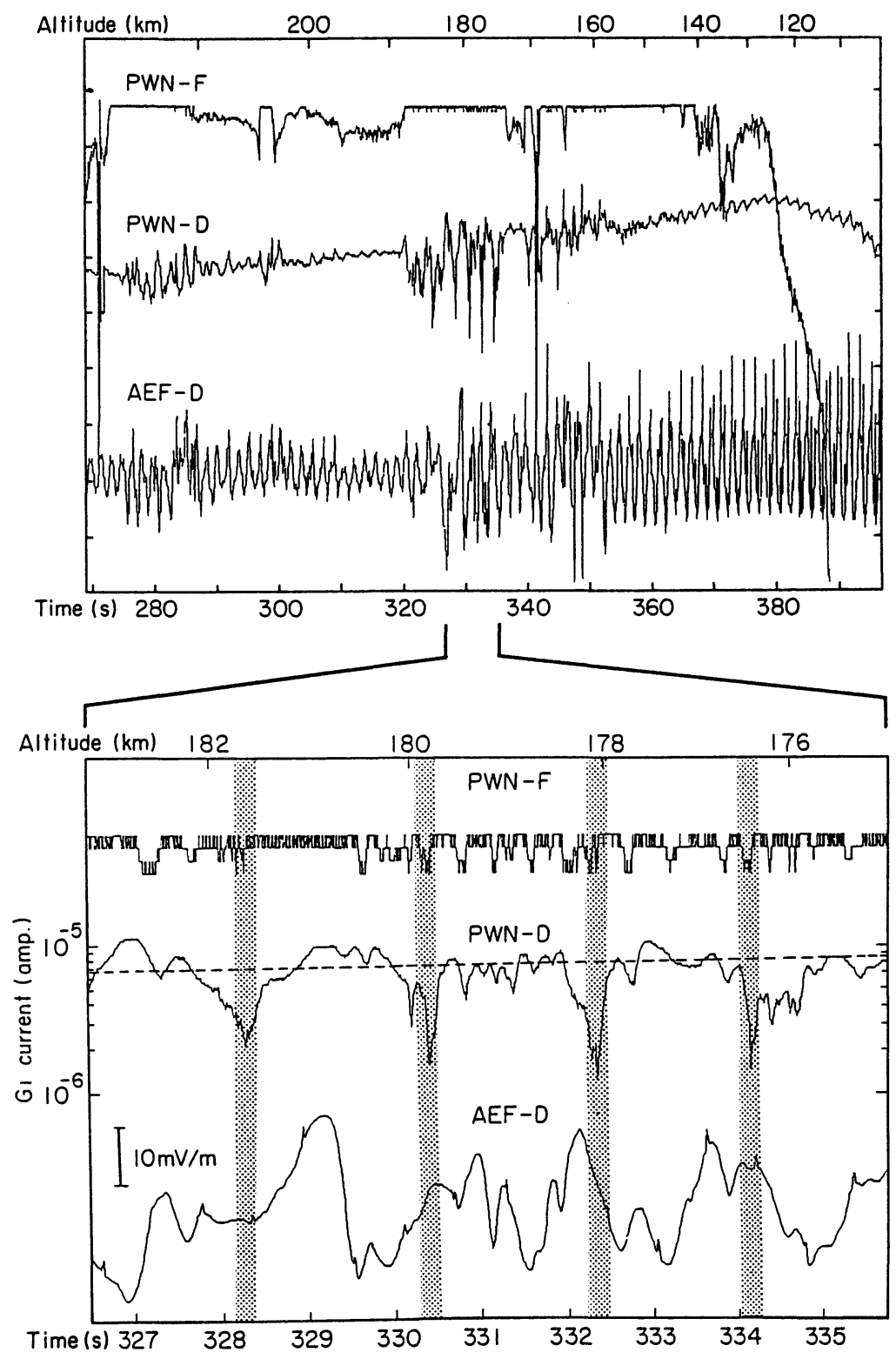

Fig. 9. Temporal variations of PWN-F, -D and AEF-D during 269-397 s (upper) and during 327-335 s (lower). 
substorm. A Faraday cup and double probes aboard the rocket observed various kinds of ELF-VLF band fluctuations. Preliminary analysis of two data sets indicates the following;

1) During the ascent, the Faraday cup observed continuous electron density fluctuations in the $E$-region and burst-like fluctuations over the region from 140 to $200 \mathrm{~km}$ altitude. The burst-like fluctuations were strongly dependent on the rocket spin phase, which indicates that they were artificially generated near the rocket.

2) Near $220 \mathrm{~km}$, quasi-sinusoidal waves with frequencies around $150 \mathrm{~Hz}$ were clearly observed by both the Faraday cup and double probes. Cross-correlation analysis indicated that they are right-handed circular or elliptically polarized waves.

3) During the descent, the rocket passed through a highly active aurora and both the Faraday cup and the double probes observed intense ELF fluctuations. The Faraday cup measurements suggest that the rocket was charged to several volts (negatively) by electron precipitation.

4) Rocket interactions with the surrounding plasma may locally excite various waves. Therefore, it is important to distinguish carefully between artificially and naturally generated plasma waves.

The rocket experiments are supported by the National Institute of Polar Research (NIPR). We would like to express our sincere thanks to the wintering party of the 26th Japanese Antarctic Research Expedition for their efforts during the rocket launchings and for providing the geophysical condition observations. We are also grateful to Profs. T. Nagata, T. Matsuda, and T. Hirasawa of NIPR for their supports for the experiments, and also to Profs. T. Oguti, I. Kimura, and M. Ejiri for their efforts in planning these rocket experiments. Also thanks are due to Dr. B. A. Whalen for his critical reading of the manuscript.

\section{REFERENCES}

Ejiri, M., H. Fukunishi, T. Ono, H. Yamagishi, T. Hirasawa, I. Kimura, and T. Oguti, Auroral phenomena observed by the sounding rockets S-310JA-8 to -12 at Syowa Station, Antarctica, J. Geomag. Geoelectr., this issue, 763-781, 1988.

Mori, H., E. Sagawa, T. Ogawa, T. Ogawa, and H. Yamagishi, Correlation analysis of electric field and electron density fluctuations observed by a sounding rocket S-310JA-7, Mem. Natl. Inst. Polar Res., Spec. Issue, 36, 238-244, 1985.

OgawA, T., H. MoRi, S. MiYAZAKI, and H. YAMAgISHI, Electrostatic plasma instabilities in highly active aurora observed by a sounding rocket S-310JA-7, Mem. Natl. Inst. Polar Res., Spec. Issue, 18, 312-329, 1981.

Ogawa, T., M. Makino, S. Hayashida, H. Yamagishi, R. Fujil, T. Hirasawa, and M. Nishino, Measurements of auroral electric fields with an Antarctic sounding rocket S-310JA-7; 1. DC electric field, Mem. Natl. Inst. Polar Res., Spec. Issue, 18, 355-378, 1981.

Takahashi, T., H. Oya, H. Fukunishi, Y. Watanabe, and H. Yamagishi, Structure of the auroral ionosphere observed by the Antarctic rockets S-310JA-11 and -12, J. Geomag. Geoelectr., this issue, 843-857, 1988.

Tohyama, F., M. Ishido, and H. Fukunishi, Attitude determination of the antarctic sounding rockets from magnetometer and horizon aspect sensor data, J. Geomag. Geoelectr., this issue, 817-829, 1988.

Yamagishi, H. and H. FukUnishi, Auroral sounding rocket experiment of the 26th Japanese Antarctic Research Expedition; mission plan, Antarctic Rec., 85, 48-61, 1985. 